How to reference this article

Pioli, M. (2020). Dalla Sicilia alla Spagna, dalla Spagna alla Sicilia: Leonardo Sciascia scrittore di viaggio. Italica Wratislaviensia, 11(2), 119-135.

DOI: http://dx.doi.org/10.15804/IW.2020.11.2.7

Marco Pioli

Universidad Complutense Madrid, Spagna

mpioli@ucm.es

ORCID: 0000-0002-8457-6626

\title{
DALLA SICILIA ALLA SPAGNA, DALLA SPAGNA ALLA SICILIA: LEONARDO SCIASCIA SCRITTORE DI VIAGGIO
}

\section{FROM SICILY TO SPAIN, FROM SPAIN TO SICILY: LEONARDO SCIASCIA AS A TRAVEL WRITER}

\begin{abstract}
The outbreak of the Spanish Civil War represented a pivotal moment in Leonardo Sciascia's ideological development, as it pushed him towards an anti-fascist passion that would make him an engagé writer over the years. In fact, the news of Lorca's assassination and Ortega y Gasset's volumes had a lasting influence on the writer: he began to read Spanish and about the Spanish world, thus discovering Spain and its language, literature, and culture. In fact, it was a rediscovery, since, in the eyes of the Sicilian author, the common Arab domination and the long Spanish hegemony in Sicily had already connected the island and the peninsula in an intricate web of "similarities." The present article aims to examine the distinctness of Sciascia's Sicilian-Spanish imaginary that is present in the reports that he published after his numerous trips to the Iberian land starting in the 1950s. After having often been dismissed as paraliterary, those works will be analysed as travel writing so as to better appreciate them. Ore di Spagna, the volume that collects most of those journalistic articles, will be considered as one of the best examples of reporting in the $20^{\text {th }}$ century, far beyond the boundaries of essay production.
\end{abstract}

Keywords: Leonardo Sciascia, Sicily, Spain, voyage, travel literature 


\section{INTRODUZIONE. \\ LEONARDO SCIASCIA IN VIAGGIO VERSO LA SPAGNA}

66 a lì comincia la nostra storia nella storia", scrisse Leonardo Sciascia a proposito della Guerra civile spagnola (1988/2000, p. 27), il cui scoppio ha rappresentato un momento decisivo per la maturazione ideologica di un'intera generazione cresciuta sotto il fascismo (Albertoni, Antonini \& Palmieri, 1962, p. 259). Precisamente, nel 1936, Sciascia aveva 15 anni e, nonostante la retorica del regime mussoliniano indicasse Francisco Franco come alleato, le notizie della presa di posizione filorepubblicana di scrittori e attori americani da lui amati, come Ernest Hemingway, Gary Cooper o Charlie Chaplin, lo condussero pian piano verso quelle posizioni antifasciste che negli anni, come dichiarò a Marcelle Padovani, lo avrebbero reso uno scrittore impegnato dalla parte “degli oppressi" (Sciascia, 1979, p. 85). Nelle Parrocchie di Regalpetra (1956) - che Sciascia considerava il suo primo vero libro (1988/2000, p. 29) - è possibile ritrovare la passione delle scelte ideologiche ispirate, in quegli anni, dalla nazione spagnola:

mi trovai dall'altra parte. Ora quei nomi delle città di Spagna mi si intridevano di passione. Avevo la Spagna nel cuore. Quei nomi - Bilbao Malaga Valencia; e poi Madrid, Madrid assediata - erano amore, ancor oggi li pronuncio come fiorissero in un ricordo di amore. E Lorca fucilato. E Hemingway che si trovava a Madrid. E gli italiani che nel nome di Garibaldi combattevano dalla parte di quelli che chiamavano rossi. [...] A pensare oggi a quegli anni mi pare che mai più avrò nella mia vita sentimenti così intensi, così puri. (Sciascia, 1956/2004, pp. 43-44)

Come risulta dal passaggio, insieme all'antifascismo Sciascia scoprì anche il suo amore per la cultura spagnola. Ben presto divenne un accanito lettore dei romanzi e dei resoconti dedicati alla Guerra civil dagli osservatori internazionali, tra i quali si segnalano La speranza di André Malraux (1937), Omaggio alla Catalogna di George Orwell (1938), I grandi cimiteri sotto la luna di Georges Bernanos (1938), Per chi suona la campana di Ernest Hemingway (1940) ed Esperienze della guerra di 
Spagna di Herbert Matthews (1946) ${ }^{1}$. Allo stesso tempo, il siciliano arrivò a confrontarsi direttamente con la cultura spagnola, iniziando a studiare il castellano da autodidatta e leggendo i classici, come El Quijote o La celestina, i saggisti di inizio Novecento - su tutti, Ortega y Gasset, Américo Castro, Eugenio d'Ors e Miguel de Unamuno - e i poeti vittime delle persecuzioni di Franco, dei quali, come nel caso di Federico García Lorca, il racalmutese fu anche un appassionato traduttore.

A questi "viaggi di carta" seguirono, poi, a partire dagli anni Cinquanta, i numerosi spostamenti che Sciascia decise di intraprendere in terra iberica, insieme alla moglie Maria Andronico. Precisamente, un primo viaggio fu programmato per il giugno 1955. Per Sciascia, già abbonatosi al Touring Club Italiano, e inseritosi nei circuiti editoriali italiani con i primi importanti lavori - tra gli altri, il volume del $1950 \mathrm{Fa}$ vole della dittatura e l'antologia Il fiore della poesia romanesca (1952), curata con Pier Paolo Pasolini -, era giunto il momento di valicare le frontiere nazionali. Tale viaggio, però, non oltrepassò i Pirenei: Sciascia preferì fermarsi nell'altro suo "luogo dell'anima", la Francia, come si deduce dallo scambio epistolare con il poeta e ispanista leccese Vittorio Bodini, recentemente raccolto da Fabio Moliterni nel volume Sud come Europa. Carteggio (1954-1960) (Bodini \& Sciascia, 2011, p. 76).

Il racalmutese raggiunge il suolo iberico esattamente un anno dopo, visitando le città di Barcellona e Madrid. Per la primavera successiva l'autore progettava una nuova trasferta, nelle terre del sud, com'è possibile riscontrare, ancora una volta, nella corrispondenza con l'amico leccese (ibid., pp. 125 e 135), mentre con maggiore esattezza è possibile segnalare un suo viaggio spagnolo nel 1966 (Sciascia, 1956/2004, pp. 5-6). Negli anni Ottanta, Sciascia tornò con frequenza nella terra di Cervantes, accompagnato anche dai fotografi Ferdinando Scianna e Giuseppe Leone (González de Sande, 2009, pp. 21-29). Si distinguono il viaggio dell'autunno del 1982, quando per un mese percorse in treno la Spagna, e quello della primavera del 1984, intrapreso per assistere alle Semanas santas delle città andaluse.

1 Si riportano le date delle prime edizioni in lingua originale. Per le ulteriori, numerose testimonianze sulla Guerra civile spagnola possedute e lette dal siciliano si rimanda allo stesso Sciascia (1988/2000, p. 29) e a Curreri (2007, pp. 49-51). 
Le esperienze itineranti appena segnalate sono piuttosto rilevanti in quanto furono alla base dei progetti editoriali attraverso i quali Sciascia impose al largo pubblico italiano il suo interesse per la cultura spagnola. Innanzitutto, i racconti Il soldato Seis (1958-1964) ${ }^{2}$ e L'antimonio, confluito quest'ultimo nella seconda edizione de Gli zii di Sicilia (1960). Entrambi i testi sono dedicati alle vicende della Guerra civil e presentano andamenti diegetici vicini ai resoconti odeporici. L'antimonio, inoltre, con la sua combinazione di contenuti autobiografici, ideologici e metaletterari, ribadisce l'importanza dell'incontro con la Spagna per la maturazione intellettuale e poetica di Sciascia, come ha accuratamente indagato Luciano Curreri (2007), il quale considera il racconto uno degli esiti "più riusciti e complessi della narrativa italiana del Novecento" (p. 14).

Tuttavia, le peregrinazioni iberiche sopra illustrate hanno incrociato anche il "secondo mestiere" di Sciascia, quello della scrittura su periodico, e hanno ripreso a "circolare" nella forma del reportage. Lo scrittore di Racalmuto, infatti, benché sia noto soprattutto per i suoi gialli atipici, si è cimentato anche con la scrittura di viaggio, ottenendo, per giunta, risultati decisamente originali, come si cercherà di dimostrare nel presente studio.

\section{LEONARDO SCIASCIA "ARTICOLISTA VIAGGIANTE"}

Alla luce dei materiali recensiti dai repertori bibliografici in circolazione (Fascia, 1998; Motta, 2009), tra i primi tentativi odeporici di Sciascia dedicati alla penisola iberica può essere segnalato il reportage "Com'era la Spagna", pubblicato su L'Ora di Palermo il 20 agosto 1966 nella rubrica Quaderno, redatta dall'autore tra il 1964 e il 1968, i cui testi furono ripubblicati in volume nel 1991 (cf. Pioli, 2019, p. 428).

2 Tale racconto, pubblicato da Sciascia per la prima volta nel 1958 sulla rivista Valbona e, dopo vari rimaneggiamenti, su Rassegna sindacale nel 1964, rappresenta una sorta di "preludio" del più ambizioso L'antimonio. Il testo si può leggere in Sciascia (1958/2012, pp. 1317-1320). 
L'occasione è offerta dal viaggio compiuto nel 1966 e l'incipit del pezzo segna immediatamente i suoi orizzonti geografici e tematici: "Barcellona, Albergo Colon: nel trentesimo anniversario dell'alzamiento" (Sciascia, 1991, p. 168). Esattamente trent'anni dopo lo scoppio della Guerra civil, Sciascia percorre le vie di Barcellona sulla scia dei ricordi bellici che avevano appassionato i suoi anni giovanili, e in questa esplorazione la guida prescelta è quella del citato romanzo di Malraux, La speranza, "forse il più grande che sia stato scritto in questi trent'anni" (1991, p. 168), le cui pagine avevano ritratto le fasi barcellonesi del conflitto. Sciascia lamenta di non aver portato il romanzo con sé, pertanto non sempre è sicuro della corrispondenza tra gli scenari di battaglia descritti dal francese e i riscontri offerti dagli spazi urbani barcellonesi, quelli degli anni Sessanta, interessati da un boom economico "all'italiana": "un qualcosa di simile a quello edilizio di Agrigento, che si sa come è andato a finire. E vien fatto di pensare che in fondo una dittatura di tipo fascista è qualcosa di molto simile a una cattiva democrazia" (1991, p. 173).

Come si evince dal passaggio appena citato, il pezzo affianca ai ricordi del triennio rivoluzionario spagnolo, essenzialmente letterari, un altro motivo ricorrente nella scrittura reportistica di Sciascia, quello del "gioco delle somiglianze" 3 con l'isola siciliana. Per esempio, probabilmente per il suo trascorso come maestro, Sciascia evidenzia che, a fronte della ripresa economica e dello sviluppo dell'ozio, in Spagna "ci sono paesi con due cinematografi e nessuna scuola. [...] Esattamente come cent'anni fa al mio paese: sorgeva uno splendido teatro comunale, ma i ragazzi andavano a scuola serale da un prete" (1991, p. 173). Persino la vista delle prostitute barcellonesi è capace di stimolare dei paragoni tra i due territori mediterranei in virtù del comune sostrato religioso: "certo è che la prostituzione è particolarmente rigogliosa nei paesi cattolici. [...] Certi paesi, in Sicilia, erano indicati - campanilisticamente, dagli abitanti di altri paesi - come di larghe vedute in fatto di morale sessuale;

${ }^{3}$ L'espressione è usata da Sciascia nell'elzeviro "L'ordine delle somiglianze", raccolto in Cruciverba (1983, pp. 989-993), per definire una consuetudine gnoseologica per lui tipica dei siciliani, cioè quella di ricercare costantemente analogie tra persone o cose. 
ed erano quelli dove clero secolare e comunità monastiche più si addensavano" (1991, p. 174). Queste riflessioni sviluppate tra la penisola e l'isola, tra il loro presente e il loro passato, non si limitano all'ambito etico-civile, ma sanno aprirsi anche a questioni artistiche e linguistiche. Non a caso, la vista delle ceramiche colorate tipiche delle architetture di Gaudí, specialmente nel Parco Güell, ricorda a Sciascia "il giuoco infantile della ricerca e disposizione decorativa dei cocci di vecchi piatti". Questi frammenti, racconta il viaggiatore siciliano, "erano chiamati 'lisciari', nell'agrigentino [...]: e non sono mai riuscito ad accertare da dove provenga il $[\ldots]$ termine (a meno che non venga appunto dallo spagnolo "lijar', far rilucere, forbire)". Alla fine di queste "impressioni", l'autore dichiara in modo alquanto significativo: "ritrovare questi [...] elementi di tradizione e di memoria, della Spagna come della Sicilia, è un fatto che mi commuove" (1991, p. 175). Con evidenza, dunque, le parole appena citate confermano che i continui "movimenti pendolari" di queste pagine - per usare la fortunata metafora proposta da Ricciarda Ricorda (2001, p. 199) - sono rivolti alla familiarizzazione dell'“estraneo spagnolo" nell'"ordine delle somiglianze" con l'isola natale o, comunque, con il patrimonio esperienziale dell'autore, e diventano, conseguentemente, la cifra distintiva dell'immaginario siculo-spagnolo sciasciano.

Proseguendo nell'esplorazione dei testi odeporici del racalmutese, secondo un criterio cronologico, occorre segnalare il ciclo di reportage redatti, a seguito del viaggio del 1982, per il Corriere della Sera, con cui Sciascia collaborava dal 1969. Tra il marzo e l'aprile 1983 compaiono quattro sue corrispondenze, le quali rientrano pienamente nei confini delle scritture periegetiche, come riprovano gli occhielli con cui il Corriere le annunciava: "Sciascia: viaggio di uno scrittore attraverso i luoghi e la cultura di Spagna" (Sciascia, 1983a); "I viaggi degli scrittori del «Corriere»: Sciascia rivisita la Spagna; Moravia racconta il suo itinerario africano" (Sciascia, 1983b e 1983c); "I viaggi degli scrittori: Sciascia in Spagna" (Sciascia, 1983d).

4 Significativamente per la loro definizione di genere, questi reportage si intrecciano con quelli di uno dei più prolifici scrittori-viaggiatori del Novecento italiano: Alberto Moravia. 
Nel primo pezzo, "A Salamanca, nell'Università di Unamuno", del 5 marzo 1983, il viaggio nella città universitaria spagnola diventa il pretesto per un altro tipo di incontro: "cerco a Salamanca il ricordo della sua agonia". Sciascia si riferisce a Miguel de Unamuno, lo storico rettore di quell'Università, del quale viene ricordato il drammatico scontro con la rivoluzione di Franco, dopo le simpatie iniziali. Tale ricordo, come al solito, riporta il siciliano in territori ben più familiari: "non è solo nell'opera di Unamuno e Pirandello che si possono trovare punti di somiglianza. Anche le loro biografie ad un punto si somigliano; e precisamente in quel loro contraddirsi e stravolgersi nell'adesione al fascismo" (Sciascia, 1983a).

I tre reportage successivi cambiano la loro "geografia" e "conducono" i lettori del Corriere nella capitale Madrid. Nel primo, pubblicato il 13 marzo 1983 e intitolato "Da Murillo ai volti dell'Inquisizione", una mostra al Museo del Prado sul pittore barocco e una sull'Inquisizione al Palacio de Velázquez, stimolano una lunga riflessione sull'altro aspetto della storia spagnola che ha sempre appassionato il racalmutese, il Santo Oficio appunto. Quest'ultimo, infatti, al pari della Guerra civile, offriva uno specchio utile per indagare quell'eterna intolleranza umana, fascista e vessatoria, di cui l'isola siciliana per Sciascia è sempre stata universale "metafora", e che nella nazione iberica continuava ad aleggiare nonostante la transizione democratica: "La Spagna ha ancora paura, [...] dall'Inquisizione - da ogni inquisizione - vuole essere finalmente libera" (Sciascia, 1983b).

Se l'incipit indica Madrid come luogo del reportage successivo, in realtà "Un gagliego al governo", del 26 marzo 1983, è dedicato a personaggi provenienti dalla Galicia. Si menziona l'incontro avvenuto con il pittore Jaime del Valle Inclán, figlio del più celebre Ramón del Valle Inclán, scrittore che per la sua indole estetizzante è avvicinato da Sciascia a D'Annunzio. Il pezzo, però, ricorda soprattutto Pio Cabanillas Gallas, ex ministro di Franco, capace di sopravvivere politicamente nella nuova democrazia spagnola, così come il siciliano Liborio Romano era riuscito a passare dal Regno borbonico al regime garibaldino per finire nel Parlamento del Regno d'Italia. Per rimarcare la sua posizione politica, alla fine Sciascia concede spazio anche all' 'immagine che tutto 
il resto della Spagna ha del galiziano [...]: di indecifrabile astuzia, di tenace vocazione a ogni tipo di potere, di infinita capacità nella mistificazione, nell'inganno. Era gagliego anche Franco" (Sciascia, 1983c).

Tuttavia, Sciascia è ben consapevole che in Spagna può essere rischioso maneggiare qualsiasi tipo di definizione regionale, come scrive nell'ultimo articolo del ciclo, "Qui un siciliano ritrova i vicerè", dell'8 aprile 1983: "Una delle più gravi gaffes, conversando con un catalano o un basco o un valenciano, è quella di parlare di regione, di regionalismo, di autonomie regionali. Bisogna parlare di nazione, nazionalismo, autonomie nazionali" (Sciascia, 1983d). Sicuro delle conoscenze empiricamente acquisite nel corso delle sue frequentazioni iberiche, l'autore divaga su alcune questioni legate ai rapporti tra la sua isola e la Spagna. In particolare, si focalizza sulla scarsa presenza di libri spagnoli nelle biblioteche siciliane e sulla pressoché inesistenza di libri sulla Sicilia scritti da autori spagnoli, se si escludono le relazioni redatte dai viceré. La memoria del viaggiatore siciliano, però, è capace di riscattare dall'oblio i secoli di storia passati in comune:

Ma nonostante tanta reciproca estraneità (un tempo forse qualcosa di peggio: insofferenza, odio), andare per la Spagna è, per un siciliano, un continuo insorgere della memoria storica, un continuo affiorare di legami, di corrispondenze [...]. E bastano i nomi: di paesi, di strade. Che sembrano sentirli risuonare, nella lontana eco del tempo, dalla voce dei banditori: il viceré duca d'Ossuna, il viceré duca di Medinaceli, il viceré duca di Maqueda, il viceré marchese di Villena... (Sciascia, 1983d)

\section{ORE DI SPAGNA, UN LIBRO DI VIAGGIO}

Sciascia raccolse gli ultimi tre articoli appena analizzati, insieme ad alcuni elzeviri e ai resoconti del viaggio spagnolo del 1984, nel volume Ore di Spagna, pubblicato nel 1988 da Pungitopo5. Il libro, che accoglie anche un reportage fotografico di Ferdinando Scianna, prese forma,

${ }^{5}$ Questi pezzi erano stati pubblicati, oltre che sul Corriere, sul Giornale di Sicilia e sul periodico Epoca. Il volume ha avuto una successiva edizione, nel 2000, presso Bompiani, dalla quale si citerà nel corso di questo studio, e un'ultima, nel 2016, presso Contrasto (Roma). 
in realtà, grazie all'iniziativa di Natale Tedesco. Tuttavia, Sciascia partecipò alla selezione dei testi e scelse sia l'editore che il titolo (Lo Cascio, 2009, p. 53; Fava Guzzetta, 1992, p. 81) ${ }^{6}$. Tale volume ha rappresentato uno svincolo importante per la critica sciasciana, in quanto ha imposto la riconsiderazione del ruolo della Spagna nel laboratorio letterario dell'autore, a scapito dei modelli francesi, da sempre ampiamente valorizzati dagli studiosi (Traina, 2000, pp. 206-209).

Rispetto ai reportage del Corriere, Ore di Spagna aggiunge nuovi itinerari geografici e culturali, anche se il patrimonio memoriale e affettivo correlato con la Guerra civil continua a essere la lente privilegiata attraverso cui leggere lo spazio iberico. La "passione ideale e libresca" (Sciascia, 1988/2000, p. 61) che ispira il viaggiatore siciliano spiega perché si ricerchino e si interroghino i luoghi delle battaglie più crudeli, come Belchite o Guadalajara, dove, nel 1937, si scontrarono gli italiani del Battaglione Garibaldi con quelli del C.T.V a fianco di Franco: "I contadini $[. .$.$] ci confermarono che eravamo a Palacio Ibarra. «Siete$ i primi italiani», ci dissero «che vengono per domandare; spesso vengono degli italiani, ma per ricordare.» Loro non ricordavano: erano venuti dopo la guerra. [...] Ma fino a pochi anni prima, nell'arare vedevano affiorare ossa di italiani" (Sciascia, 1988/2000, p. 67).

La centralità delle tematiche storico-politiche emerge anche quando Sciascia si confronta con contesti più vicini alla dimensione folkloristica. Come durante la visita a Palmar de Troya - dove l'antipapa Gregorio XVII eresse il suo piccolo Vaticano, dal quale poi proclamò la beatificazione di Franco - o quando assiste alle Semanas santas andaluse: "E a Siviglia, stando al centro della città, [...] viene da pensare che il generale Queipo de Llano si sia servito dell'itinerario che le processioni percorrono nella Semana Santa per impadronirsi subito, la stessa notte dell'alzamiento dei militari, della città" (Sciascia, 1988/2000, pp. 54 e 47).

${ }^{6}$ Diversamente è avvenuto con il recente Parigi (Sciascia, 2020), in cui le fotografie di Scianna accompagnano quattro brevi reportage di Sciascia selezionati da Paolo Squillacioti. 
Allo stesso tempo, la menzionata natura "libresca" dell'interessamento sciasciano per la Guerra civile spiega perché questa continui a essere ricordata attraverso la letteratura - alla quale comunque Sciascia ha sempre riservato un evidente primato ermeneutico sulla realtà (Onofri, 1994, pp. 221-249). Agli autori già menzionati - Malraux, Hemingway, Orwell, Bernanos, ecc. - si affiancano i nomi dei tanti scrittori spagnoli che si sono dovuti confrontare, o propriamente scontrare, con il franchismo, come i citati Unamuno e Lorca, Antonio Machado, Pedro Salinas, Jorge Guillén, Luis Cernuda e Manuel Azaña, ai quali occorre aggiungere il cileno Pablo Neruda (Sciascia, 1988/2000, pp. 29, 35 e 69-71).

Data questa premessa, non sorprenderà che il viaggiatore indugi con lunghe riflessioni sul Don Quijote ad Alcalà de Henares, la città natale di Cervantes, o nella Mancha, "con la sua terra rossa, le viti basse, i bianchi mulini a vento sulle creste delle colline, le osterie in cui si ritrovano sapori che appartengono a lontane infanzie [...] che ci riportano alle soste rifocillanti di don Chisciotte e Sancio" (1988/2000, pp. 41-42). Ore di Spagna rende omaggio, allora, a uno degli autori spagnoli che maggiormente segnarono la maturazione intellettuale di Sciascia, sia in direzione dell'apprendimento linguistico - "sulle Obras di Ortega ho appreso quel po' di spagnolo che so" - sia in direzione di una visione "adulta y ordenada del mundo contemporáneo" (González Martín, 2000, p. 744). Si tratta chiaramente di Ortega y Gasset, le cui Obras completas furono scoperte dal giovane Sciascia "in una bottega di vecchi libri" e lette secondo una modalità coerente con le prospettive critiche della presente analisi:

debbo dire di non averle lette e rilette, quelle pagine, come di un filosofo. L'affermazione può sembrare estravagante e paradossale: ma le Obras di Ortega erano per me come un grande libro di viaggio, un viaggio straordinario, avventuroso, ricco di imprevisti e di rivelazioni nelle regioni dell'intelligenza. (Sciascia, 1988/2000, p. 33)

Difatti, anche Ore di Spagna può essere considerato un libro di viaggio. Nonostante sia spesso ascritto alla produzione saggistica del 
racalmutese ${ }^{7}$, il volume, in realtà, si avvicina alla definizione di "reportage narrativo" proposta da Nicola Bottiglieri (2001, p. 38):

I reporter scrivono articoli che spesso somigliano a souvenir comprati o raccolti in un luogo per conto del lettore; poi quando questi articoli sono diventati numerosi, allora si può pensare alla trasformazione di molti reportage in un reportage narrativo, anche se per arrivare a questo è necessaria una strategia, un progetto diegetico letterario, insomma una idea forte che inglobi tutto il materiale raccolto. Gli appunti, gli articoli già scritti, le foto fatte possono servire da "intertesto" nei confronti del "macrotesto" che è il reportage narrativo. Il risultato di questi libri, nati spesso riconfezionando articoli di giornale, è una scrittura che mescola insieme linguaggi diversi: il saggio, l'articolo, l'intervista, il racconto breve, la descrizione di costume, spesso anche foto o cartine geografiche $[\ldots]$.

Ore di Spagna aspira a configurarsi come libro di viaggio già nell'ordinamento dei pezzi, in cui la coerenza tematica e geografica vince sulla cronologia della composizione. Tale organizzazione riflette le intenzioni editoriali di Tedesco - attento a selezionare quelle pagine sciasciane che "per la loro qualità di elzeviri e di letteratura odeporica" potessero conferire "al volume un'unità che non avrebbe se avessi riunito altri scritti", come si legge nella nota al testo (2000, p. 7) - e garantisce l'autonomia del nuovo prodotto editoriale rispetto agli articoli iniziali, secondo una prassi tipica del genere (Meda, 2011, p. 186). La serie, inoltre, viene inaugurata dal reportage apparso sul Corriere il 13 marzo 1983, il quale esordiva con una riflessione sull'andare che può servire da bussola orientativa anche per i lettori del libro:

Il giusto viaggiare è quello di non conoscere, nei luoghi in cui si va, nessuna persona o pochissime; di non avere commendatizie da consegnare e appuntamenti cui consegnarsi; di non avere impegni che con se stessi, per vedere senza affanni le cose che abbiamo desiderato vedere e che di solito, almeno per me, non sono - di una regione, di una città - moltissime. Certo l'incontro

7 Come sembrerebbe confermare la sua collocazione nel tomo Saggi letterari, storici e civili (2019) della nuova edizione delle Opere di Adelphi, la quale ha il grande merito di includere il libro qui in analisi, rimasto invece fuori dalla precedente edizione Bompiani delle Opere complete. 
con persone può anche funzionare come rivelazione di quello che si suol dire il genio del luogo; ma oggi un po' meno ed è comunque meglio contemplare un tal genio nelle cose. Trent'anni fa viaggiavo con molta libertà e con grande piacere; oggi, nella trama degli incontri e degli impegni, con poca libertà e minimo piacere. (Sciascia, 1988/2000, p. 21)

Una sintesi di queste considerazioni, tra l'altro, accompagna l'immagine di copertina dell'edizione spagnola del volume, il quale può vantarsi pure di una successiva versione francese (Sciascia, 1992). Si legge nella traduzione di Carlos Manzano: "La forma cabal de viajar es la de no tener cartas de recomendación ni citas, no tener compromisos sino con uno mismo" (Sciascia, 1990). Questa soluzione dell'editore spagnolo orienta fortemente il volume verso la letteratura di viaggio, genere che si avvale anche del paratesto, secondo Luca Clerici (1996, p. 805), ai fini della definizione dei suoi confini editoriali, per statuto incerti e ancora più labili nel Novecento. Se nelle edizioni italiane di Ore di Spagna non si trovano indicazioni di copertina così esplicite, comunque rimangono a sostenere la presente "interpretazione odeporica" le fotografie di Scianna, le quali costituiscono parte integrante del progetto.

Lo stesso Clerici, in effetti, ha parlato di una "vocazione del libro di viaggio a essere accompagnato dall'immagine" (2013, p. XXV), vocazione che dopo essersi servita delle stampe xilografiche si è espressa compiutamente con l'affermazione della fotografia. Le immagini decorano il volume, sanciscono la fattualità dello spostamento e trasmettono messaggi culturali. Nel Novecento, a partire almeno dagli anni Trenta, il libro di viaggio propone la formula che abbina le parole di scrittori affermati con le immagini di grandi fotografi, e proprio alla luce di questa tradizione - tutta da studiare (Clerici, 2013, p. XXVI) - sarebbe opportuno valutare la fusione editoriale istauratasi tra le parole di Sciascia e gli scatti di Scianna.

Se il numero di questi scatti è cambiato tra un'edizione e l'altra di Ore di Spagna, tale incombenza non delegittima la presente lettura del volume, in quanto è nello statuto del genere una certa fluidità dei paratesti, con casi, addirittura, di passaggi di materiali da un libro a un altro (Adamo, 1999, p. 539). Tali fotografie, inoltre, sono state scattate da Scianna durante i viaggi condivisi con l'amico scrittore e spesso ri- 
traggono personaggi, situazioni e luoghi della Spagna comunicanti con i temi evocati dai testi del volume, compensando così quel modo di guardare "senza macchina fotografica" che rappresenta la peculiarità, come si sarà percepito, della scrittura periegetica di Sciascia.

\section{L'ODEPORICA DI SCIASCIA: UN VIAGGIO NEL TEMPO}

L'odeporica del racalmutese è sicuramente lontana dai classici della Reiseliteratur, poiché in essa l'ordo argomentativo vince sulla narrazione e soprattutto sulla descrizione, secondo una prassi tipica anche della produzione maggiore dell'autore. L'intonazione è memoriale, "elzeviristica", trascura l'hic et nunc della composizione, e pochi sono anche gli incontri. Un lettore avvezzo ai resoconti di viaggio dedicati alla Spagna, spesso vissuta come "culla dell'esotico europeo" (De Pascale, 2001, p. 74), resta sorpreso soprattutto della scarsa attenzione riservata al folklore iberico, il quale, invece, con il suo repertorio di toros, sivigliane, vino e chitarre, ha rappresentato un'attrattiva per i viaggiatori di ogni epoca e, di conseguenza, un tema testualmente molto produttivo ${ }^{8}$.

Come anticipato, sono le menzionate foto di Scianna a completare queste carenze o vuoti descrittivi, specialmente mediante alcuni scatti dedicati a situazioni topiche, quali una corrida sevillana, delle contadine al lavoro, alcune taverne, una gitana mendicante o un cieco rivenditore di lotterie, seppur con prospettive mai banali. Il bianco e nero di Scianna, attento a restituire la dimensione socio-antropologica della terra percorsa, sembra riaffermare, infatti, il punto di vista del viaggiatore Sciascia, per la cui definizione possono essere d'aiuto le seguenti riflessioni di Walter Benjamin (1971, p. 101):

Lo stimolo epidermico, l'esotico, il pittoresco prendono solo lo straniero. Ben altra, e più profonda, è l'ispirazione che porta a rappresentare una città nella prospettiva di un nativo. È l'ispirazione di chi si sposta nel tempo invece che nello spazio. Il libro di viaggi scritto dal nativo avrà sempre affinità col libro di memorie: non invano egli ha vissuto in quel luogo la sua infanzia.

8 Sulle tracce di questo immaginario nella cultura occidentale moderna e contemporanea si veda Santos Unamuno (1997). 
Benché non si abbia a che fare con un "nativo", anche il legame di Sciascia con la Spagna è altrettanto genetico. Riprendendo il reportage dell'8 aprile 1983, confluito nel cap. IX di Ore di Spagna, e integrando il passaggio solo parzialmente citato in precedenza, si legge che il "continuo insorgere della memoria storica", implicato dall'"andare per la Spagna", è dovuto - nelle parole dell' autore (2000, p. 60) - "a qualcosa di simile $[\ldots]$ a una ritrovata fraternità. E dico ritrovata pensando allo splendido dominio degli arabi che Spagna e Sicilia ebbero comune".

Sciascia, dunque, non può percorrere la Spagna come un estraneo, in quanto si orienta grazie al passato civile e culturale indagato, tramite i vagabondaggi letterari, a partire dalla passione intellettuale scatenata dalla Guerra civil. Inoltre, nel suo immaginario, la dimensione siciliana, così vicina alla Spagna per ragioni storiche, si impone come costante motivo di confronto analogico. La sua ricerca del "gioco delle somiglianze" tra l'isola e la penisola, nonostante comporti un'evidente elusione della diversità spagnola, riesce perciò a generare un'interpretazione dell'altrove iberico profonda e personale, lontana dai noti cliché e dalla consueta oleografia turistica. In definitiva, tale tensione assimilativa - avvertibile anche in molte delle fotografie di Scianna - rappresenta quel "principio primo catalizzante e ordinatore di tutta la materia" (Farnetti, 1994, p. 7) capace di uniformare un volume come Ore di Spagna in direzione dei migliori esiti reportistici novecenteschi.

Difatti, innanzi alla massificazione e alla "riproducibilità tecnica" dello spostamento, la letteratura di viaggio può riscattare la sua capacità di significare l'alterità solamente imponendo punti di vista non omologati, che sacrifichino "i diritti del colore" e perseguano una sovrapposizione sull'elemento spaziale della dimensione temporale, quella del vissuto del traveller o della storia dei popoli (Debenedetti, 1971, p. 328). I reportage sciasciani, richiamando le isole della memoria in un arcipelago odeporico confinante con la saggistica, sembrano conformarsi con questa buona pratica, confermata in ultima istanza dal titolo del volume che ne riunisce i risultati migliori, Ore di Spagna appunto. In esso, il referente geografico incrocia la dimensione temporale e la peculiare idea di Spagna che si genera costituisce il definitivo garante di quell'unità formale ricercata da Tedesco, consentendo così al libro di superare 
i confini del semplice compendio saggistico e di indirizzare i suoi contenuti, finalmente, "in un destino di verità".

\section{BIBLIOGRAFIA}

Adamo, S. (1999). L'esotico illustrato. Libri di viaggio in Russia e in Spagna tra Otto e Novecento. In G. N. Ricci (Ed.), Immagine, segno, parola: processi di trasformazione (vol. 2, pp. 539-560). Milano: Giuffrè.

Albertoni, E. A., Antonini, E., \& Palmieri, R. (Eds.). (1962). La generazione degli anni difficili. Bari: Laterza.

Benjamin, W. (1971). Immagini di città. Torino: Einaudi.

Bodini, V., \& Sciascia, L. (2011). Sud come Europa. Carteggio (1954-1960) (edited by F. Moliterni). Nardò: Besa.

Bottiglieri, N. (2001). L'esperienza del viaggio nell'epoca della sua riproducibilità narrativa. In N. Bottiglieri (Ed.), Camminare scrivendo. Il reportage narrativo e dintorni (pp. 7-47). Cassino: Edizioni dell'Università degli studi di Cassino.

Clerici, L. (1996). La letteratura di viaggio. In F. Brioschi, \& C. Di Girolamo, Manuale di letteratura italiana. Storia per generi e problemi (vol. IV, pp. 778-805). Torino: Bollati Boringhieri.

Clerici, L. (2013). La civiltà del viaggio. In L. Clerici (Ed.), Scrittori italiani di viaggio 1861-2000 (pp. XI-XXXVII). Milano: Mondadori.

Curreri, L. (2007). Le farfalle di Madrid. L'antimonio, i narratori italiani e la guerra civile spagnola. Roma: Bulzoni.

De Pascale, G. (2001). Scrittori in viaggio. Narratori e poeti italiani del Novecento in giro per il mondo. Torino: Bollati Boringhieri.

Debenedetti, G. (1971). Il romanzo del Novecento: quaderni inediti. Milano: Garzanti.

Farnetti, M. (1994). Reportages. Letteratura di viaggio del Novecento italiano. Milano: Guerini.

Fascia, V. (Ed.). (1998). La memoria di carta: bibliografia delle opere di Leonardo Sciascia. Milano: Otto/Novecento.

Fava Guzzetta, L. (1992). Avevo la Spagna nel cuore. In L. Fava Guzzetta (Ed.), Nelle regioni dell'intelligenza: omaggio a Leonardo Sciascia (pp. 81-86). Marina di Patti: Pungitopo.

González de Sande, E. (2009). Leonardo Sciascia e la cultura spagnola. Catania: La Cantinella. 
González Martín, V. (2000). España en la obra de Leonardo Sciascia. Cuadernos de Filología Italiana, $n^{\circ}$ extra. 1-2, 733-756.

Lo Cascio, V. (2009). Sciascia, un classico irregolare (intervista a Natale Tedesco). In V. Lo Cascio (Ed.), Verità e giustizia. Leonardo Sciascia vent 'anni dopo (pp. 46-61). Milano: Academia Universa Press.

Meda, A. (2011). 'Articolismo viaggiante'. Evoluzioni primonovecentesche del genere odeporico. Studi e problemi di critica testuale, 83, 179-196.

Motta, A. (Ed.). (2009). Bibliografia degli scritti di Leonardo Sciascia. Palermo: Sellerio.

Onofri, M. (1994). Storia di Sciascia. Roma-Bari: Laterza.

Pioli, M. (2019). L'immaginario spagnolo di Leonardo Sciascia: genealogie mediterranee. Italian studies, 74(4), 427-441.

Ricorda, R. (2001). L'andare per la Spagna di un siciliano: immagini di viaggio. In N. Tedesco (Ed.), Avevo la Spagna nel cuore (pp. 191-207). Milano: La Vita Felice.

Santos Unamuno, E. (1997). Italia e Spagna tra folklore e canzonetta. In D. Puccini (Ed.), Gli spagnoli e l'Italia (pp. 281-284). Milano: Scheiwiller.

Sciascia, L. (1979). La Sicilia come metafora (intervista di M. Padovani). Milano: Mondadori.

Sciascia, L. (1983a, 5 marzo). A Salamanca, nell’Università di Unamuno. Corriere della Sera, 3.

Sciascia, L. (1983b, 13 marzo). Da Murillo ai volti dell'Inquisizione. Corriere della Sera, 3.

Sciascia, L. (1983c, 26 marzo). Un gagliego al governo. Corriere della Sera, 3.

Sciascia, L. (1983d, 8 aprile). Qui un siciliano ritrova i viceré. Corriere della Sera, 3.

Sciascia, L. (1990). Horas de España (translated by C. Manzano). Barcelona: Tusquets.

Sciascia, L. (1991). Quaderno. Palermo: Nuova Editrice meridionale.

Sciascia, L. (1992). Heures d'Espagne (translated by M. Darmon). Paris: Fayard.

Sciascia, L. (2000). Ore di Spagna (introduction by N. Tedesco, photos by F. Scianna). Milano: Bompiani (I ed. 1988).

Sciascia, L. (2004). Le parrocchie di Regalpetra. In Opere 1956-1971 (edited by C. Ambroise, pp. 1-170). Milano: Bompiani (I ed. 1956).

Sciascia, L. (2004). Cruciverba. In Opere 1971-1983 (edited by C. Ambroise, pp. 965-1282). Milano: Bompiani (I ed. 1983). 
Sciascia, L. (2012). Il soldato Seis. In Opere (edited by P. Squillacioti, vol. 1, pp. 1317-1320). Milano: Adelphi (I ed. 1958).

Sciascia, L. (2012). Gli zii di Sicilia. In Opere (edited by P. Squillacioti, vol. 1, pp. 37-249). Milano: Adelphi (I ed. con L'antimonio 1960).

Sciascia, L. (2019). Saggi letterari, storici e civili. In Opere (edited by P. Squillacioti, vol. 2, t. 2). Milano: Adelphi.

Sciascia, L. (2020). Parigi (edited by P. Squillacioti, photos by F. Scianna). Milano: Henry Beyle.

Tedesco, N. (2000). Avevo la Spagna nel cuore (Sciascia e la Spagna). In L. Sciascia, Ore di Spagna (pp. 5-17). Milano: Bompiani.

Traina, G. (1999). Leonardo Sciascia. Milano: Bruno Mondadori.

Riassunto: Lo scoppio della Guerra civile spagnola rappresentò un momento decisivo per la maturazione ideologica di Leonardo Sciascia, in quanto le sue vicende lo spinsero verso quella passione antifascista che nel tempo lo avrebbe reso uno scrittore engagé. Tuttavia, dal triennio rivoluzionario arrivarono anche le notizie dell'assassinio di Lorca, i volumi di Ortega y Gasset - su cui Sciascia iniziò a leggere il castellano, e poi il mondo -, dunque la scoperta della Spagna, della sua lingua, della sua letteratura e della sua cultura. In realtà, si sarebbe trattato di una riscoperta, poiché, agli occhi dell'autore, la comune dominazione araba e la lunga egemonia spagnola in Sicilia avevano già saldato l'isola alla penisola in un intricato intreccio di "somiglianze". Il presente studio intende esaminare le peculiarità di questo immaginario siculo-spagnolo attraverso una presentazione dei reportage pubblicati da Sciascia a seguito dei numerosi viaggi intrapresi in terra iberica a partire dagli anni Cinquanta. Trattandosi di pagine spesso allontanate dal genere che meglio può valorizzarle, si procederà, infine, a una loro valutazione in chiave odeporica e si stabilirà che Ore di Spagna, il volume che raccoglie la maggior parte di quei pezzi giornalistici, può superare i confini del compendio saggistico nella direzione dei migliori esiti reportistici novecenteschi.

Parole chiave: Leonardo Sciascia, Sicilia, Spagna, viaggio, scrittura di reportage 